\title{
EL CONCEPTO DE SIMETRÍA \\ Y LOS PRINCIPIOS DE CONSERVACIÓN \\ DE LA MECÁNICA
}

Julián Urrea B. ${ }^{*}$

\begin{abstract}
A deeper comprehension of the formal relation among symmetries of laws of nature and rules of conservation of physical quantities has become a fundamental step toward construction of concepts and prediction of experimental results, at the level of an introductory course of mechanics.
\end{abstract}

\section{RESUMEN}

Una comprensión más profunda de la relación formal que existe entre las simetrías de una ley de la naturaleza y la conservación de una cantidad física es fundamental en la construcción de conceptos y en la predicción de resultados experimentales, a nivel de un curso introductorio de mecánica.

\section{INTRODUCCIÓN}

Las líneas espectrales de la luz procedente de estrellas lejanas y de galaxias aún más distantes coinciden con las líneas espectrales observadas en experimentos sobre la tierra. Si estas ondas de luz han viajado desde los primeros instantes del universo hasta hoy, el espectro emitido por un elemento, el hidrógeno, por ejemplo, hace 10 millones de años, es el mismo que observamos hoy en el laboratorio a partir del mismo elemento.

Así mismo el resultado de una medición de laboratorio es independiente de la hora del día o de la noche en que un evento es observado, de modo que circunstancias como el tiempo, la posición, la orientación y el estado de movimiento del observador no modifican una ley física. El Universo es, posiblemente, el mismo siempre y en todas partes: es homogéneo e isotrópico y la certeza que nos ofrecen los experimentos, sin ser absoluta, nos ayuda a entender la naturaleza en términos de un sistema coherente de leyes físicas.

\section{CONCEPTOS BASICOS}

Con el nombre de constantes del movimiento se designan ciertas cantidades físicas que, dependiendo de magnitudes como coordenadas y momentos, conservan su valor inicial, independientemente de la variación de coordenadas y momentos durante el movimiento. La conservación del momento lineal durante un desplazamiento rectilíneo, de la energía mecánica total y del momento angular de sistemas aislados, se explica a partir de la homogeneidad del espacio, de la uniformidad del tiempo y de la isotropía del espacio, respectivamente.

\footnotetext{
*Profesor Asociado. Universidad Pedagógica Nacional.

Grupo de investigación "LA ENSEÑANZA DE LA FÍSICA Y LA RELACIÓN FÍSICA-MATEMÁTICAS", Departamento de Física, Facultad de Ciencia y Tecnología, U.P.N.
} 
Comúnmente la palabra simetría significa armonía entre las partes y el todo y en sentido amplio es "una idea a través de la cual el hombre, en todos los tiempos, ha tratado de captar y de crear el orden, la belleza y la perfección" ${ }^{1}$. En el lenguaje propio de la geometría, la idea de simetría puede describirse como la propiedad de una configuración espacial según la cual un grupo de transformaciones que se aplican a la configuración la transforman en sí misma.

En lo que hace referencia a las leyes de la naturaleza la simetría tiene qué ver con hechos como que la traslación en el espacio no altera un fenómeno físico o que ante una variación en el tiempo las leyes físicas permanecen inalteradas.

Podemos ilustrar el caso de las simetrías de una figura geométrica como es el triángulo equilátero (Fig. 1). Consideremos las transformaciones siguientes:

Tres rotaciones en el plano alrededor de un eje que pasa por su centro geométrico, en ángulos de $360^{\circ}, 120^{\circ}$ y $240^{\circ}$ y tres reflexiones con respecto a los ejes a, b y c (Fig. 3). Las transformaciones del triángulo equilátero que dan como resultado una figura indistinguible de la figura original reciben el nombre de operaciones de simetría.

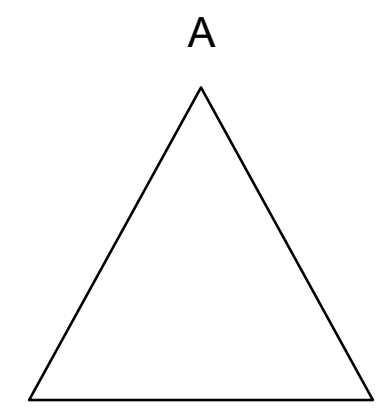

B

Figura 1

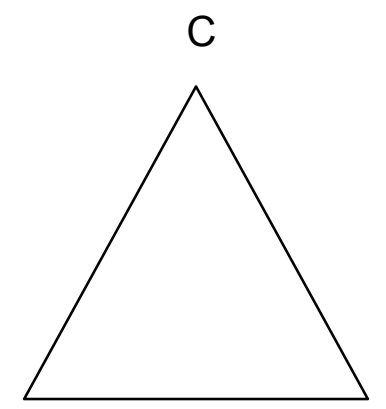

A

Figura 2

Una rotación en ángulo de $360^{\circ}$ (i) reproduce la configuración inicial en tanto que una rotación en ángulo de $120^{\circ}\left(r_{120}\right)$ cambia su orientación, como se ve en la figura 2 . Algo semejante ocurre para una rotación en ángulo de $240^{\circ}\left(R_{240}\right)$. La reflexión con respecto al eje a $\left(S_{a}\right)$ intercambia los vértices $B$ y $C$ y un efecto análogo resulta de las reflexiones de la figura con respecto a los ejes b y c.

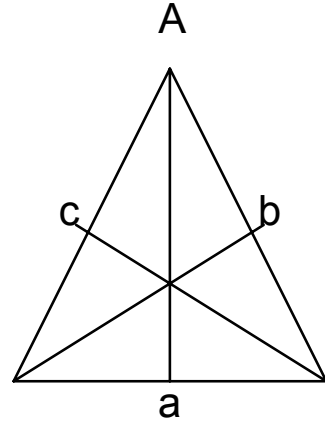

B

Figura 3

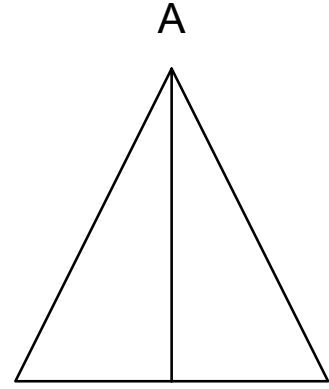

C

Figura 4

\footnotetext{
${ }^{1}$ H. Weyl. Simetría. En: El Mundo de las Matemáticas, J. R. Newman (Ed.), Grijalbo, Barcelona, 1979, vol. 4, pág. 269.
} 
El conjunto $G$ de operaciones de simetría:

$G=\left\{i, r_{120}, r_{240}, S_{a}, S_{b}, S_{c}\right\}$

Se caracteriza por el hecho de que el resultado de dos operaciones consecutivas cualesquiera del mismo es igual al resultado de una sola operación del conjunto. Por ejemplo, si el punto (.) es el signo de multiplicación de elementos de G y "multiplicar" significa la aplicación consecutiva de derecha a izquierda de las operaciones de simetría

$R_{120}$ y $s_{a}$ se obtiene:

$r_{120 .} s_{a}[A, B, C]=[B, A, C]=s_{c}[A, B, C]$.

Lo que equivale a afirmar que:

$r_{120} \cdot s_{a}=s_{c}$

Intercambiando el orden de las operaciones anteriores tenemos:

$s_{a} \cdot r_{120}=S_{b}$

Como puede verse, en general, la operación de multiplicación (.) no es conmutativa. Sin embargo el resultado es también una operación de simetría de G como se mencionó antes. Del mismo modo, la multiplicación de cualquier elemento $x$ de $G$ por $r_{360}=\mathrm{i}$ (elemento idéntico) es igual a $x$ :

i. $x=x$

También es fácil verificar que para cada operación de simetría $x$ de $G$ hay otra operación y, del mismo conjunto que anula el efecto de la primera, es decir:

$x \cdot y=i$

Se dice que $\mathrm{G}$, conjuntamente con la operación de multiplicación (.), tiene estructura de grupo, o simplemente que es un grupo.

\section{Simetrías y leyes de conservación}

La importancia fundamental de las simetrías en el estudio de la Física radica en que "para cada una de las reglas de simetría hay una ley de conservación correspondiente: hay una conexión definida entre las leyes de conservación y las simetrías de las leyes físicas"2.

Como en el caso del triángulo equilátero, en que una operación de simetría conserva su configuración geométrica, una operación realizada sobre un experimento de Física, tal que el resultado del experimento no se altera, es también una operación de simetría. Si, además, esta operación puede realizarse sobre cualquier experimento imaginable, es una operación de simetría de una ley física.

\footnotetext{
${ }^{2}$ R. Feynman. Física. Addison-Wesley Iberoamericana, Wilmington, 1987, vol. 1, págs.52-5.
} 
A manera de ejemplo consideremos un experimento de medición de la masa del electrón, que se realiza en un laboratorio en el que es posible variar las siguientes configuraciones:

-Posición el en espacio

-Orientación

- Tiempo en que se realiza la medición.

- Velocidad del laboratorio ${ }^{3}$.

La medición de la masa del electrón se realiza una y otra vez con el fin de establecer la dependencia del resultado de las distintas configuraciones del laboratorio. Sin embargo, dentro de un alto grado de precisión de la medición, se halla que el valor obtenido es completamente independiente de la posición, de la orientación, del tiempo y de la velocidad del laboratorio.

En este sentido las operaciones de translación y de rotación en el espacio, de translación en el tiempo y de substitución de una velocidad constante del laboratorio por otra, son operaciones de simetría de una ley física. Si se establece, finalmente, esta independencia de los resultados para la medición de la velocidad de la luz, de la carga del electrón, de la constante de Planck y de la constante de la gravitación universal de Newton, con respecto a los mismos parámetros, entonces las operaciones antes mencionadas son, en general, operaciones de simetría de las leyes físicas.

De hecho las simetrías relacionadas con la posición, la orientación, el tiempo y la velocidad, forman el grupo de Poincaré, el grupo espacio-temporal fundamental de las leyes de la naturaleza, simetrías que son válidas tanto a escalas cosmológicas como a nivel de la mecánica cuántica.

Más allá de este hecho fundamental, como se indicó al comenzar la presente sección, existe una relación entre las simetrías del espacio-tiempo y una clase particular de leyes físicas, como lo establece el Teorema de Noether ${ }^{4}$ :

Por cada simetría continua de las leyes de la Física hay una correspondiente ley de conservación.

La idea de conservación de una cantidad física es una idea afín a la idea de simetría: por ejemplo, las leyes de conservación más conocidas que se aplican a sistemas aislados, a saber, ley de conservación del momento lineal, ley de conservación de la energía y ley de conservación del momento angular, corresponden a las simetrías de traslación en el espacio y en el tiempo y a la simetría de rotación en el espacio.

Esta correlación entre simetría y ley de conservación determina la naturaleza de la cantidad que se conserva en función de las características de la simetría de la ley física. Las operaciones de simetría de translación pueden efectuar-se en una dirección cualquiera del espacio tridimensional, por lo tanto la cantidad física a que se refiere la ley de conservación correspondiente, el momento lineal, es una cantidad vectorial. Otro tanto ocurre con el vector momento angular y con las posibles rotaciones alrededor de los tres ejes del mismo sistema.

\footnotetext{
${ }^{3}$ C. Kittel y W Knight. Mecanics. Berkeley Physics Course, vol. 1, Chap. 3, McGraw-Hill, New York, 1965.

${ }^{4}$ C T. Hill. Symmetry in Physics. In Topics in Modern Physics National Institute, Fermilab, 1992, vol. 3, pág. 77.
} 
Por otra parte, la conservación de la energía está relacionada con la simetría de translación en el tiempo a lo largo de un eje único y por lo tanto, en el dominio de la física clásica, es una cantidad escalar. La relatividad, en cambio, bajo el sistema único espaciotiempo, reúne energía y momento lineal en una sola cantidad: el cuadrivector momentoenergía que es un invariante relativista en el espacio-tiempo ${ }^{5}$.

También se cumple la ley de conservación de la masa, de la carga eléctrica y de cantidades menos conocidas, como el número bariónico; aunque el teorema de Noether se aplica también en estos casos, la conexión con la correspondiente simetría de la ley física se da a un nivel más profundo cuya comprensión por parte de los físicos de hoy ha sido, precisamente, un salto definitivo hacia los más recientes descubrimientos de la Física Moderna.

\section{CONCLUSIÓN}

A quien interese indagar acerca de la realidad, más allá de observar la simetría de los objetos de la naturaleza, le corresponde entender la simetría de las leyes del movimiento. Pues si bien la singularidad de su experiencia está lejos de los principios generales y la comprensión de una ley no conlleva indisolublemente a la explicación de un fenómeno, la simetría de las leyes lo sitúan más cerca de las constantes en la evolución de un sistema y de la comprensión de los elementos esenciales del conocimiento del mundo.

Una explicación de los principios de conservación de la mecánica en el aula de clase, a partir de las simetrías fundamentales del espacio y del tiempo, facilitará al estudiante la identificación de la velocidad constante en línea recta, como otra forma de simetría de las leyes físicas en conexión con los sistemas inerciales de la relatividad especial.

\footnotetext{
${ }^{5}$ D. Klepner and R. J. Kolenkov. An Introduction to Mechanics. McGraw-Hill, Aukland, 1982, pág. 527.
} 